Biol. Cybern. 50, 9-13 (1984)

\title{
Characterization of Discrete and Continuous Modes of Visual Pattern Discrimination
}

\author{
Mario Ferraro ${ }^{\star}$ and David H. Foster \\ Department of Communication and Neuroscience, University of Keele, Keele, Staffordshire ST 5 5BG, England
}

\begin{abstract}
Discrete and continuous modes of visual pattern discrimination performance are analyzed using a model for the investigation of discrete internal pattern representations described in previous papers (Foster, 1980a, b). A simple quantitative criterion is derived to characterize the two kinds of visual discrimination performance. Values predicted by this criterion are then compared with values obtained from experimental data.
\end{abstract}

\section{Introduction}

Data from several experimental paradigms show that discrimination performance for shape or arrangement of simple visual patterns depends critically on factors such as the number of pattern components or subpatterns presented in the stimulus field and display duration. When visual attention is distributed over several subpatterns (typically four or more) in a display and display duration is short, visual discrimination seems to be determined by coarse, discrete encoding processes. Such performance has been reported in the discrimination of simple 3-dot figures when their deviation from collinearity is varied systematically (Foster, 1979, 1982) and in the discrimination of curved lines as curvature is varied systematically (Foster, 1983). Discrete modes of performance have also been observed in low-level perception tasks involving grouping effects with multiple arrays of geometric elements forming textures (see, for example, Olson and Attneave, 1970; Julesz, 1971, 1980; Beck, 1972; Beck and Ambler, 1972, 1973; Fox, 1978). In contrast, when the number of subpatterns in the visual field is reduced and prolonged viewing is permitted,

\footnotetext{
* On leave from Istituto di Fisiologia e Chimica Biologica, Università di Torino, Italy
}

visual discrimination appears to be determined by smooth, continuous processes (Foster, 1982, 1983). This situation is characteristic of conventional acuity and hyperacuity performance (Ludvigh, 1953; Westheimer and McKee, 1977; Westheimer, 1979, 1981). In the present paper these two kinds of discrimination performance are analyzed in terms of a model proposed by Foster $(1980 \mathrm{a}, \mathrm{b})$ in an investigation of discrete internal representations assumed to be generated by the visual system in response to patterned stimulation. A simple objective criterion to characterize the two kinds of discrimination performance is proposed and its predictions are compared with observed performance.

\section{Discrete Internal Representations and Pattern Perturbations}

An introduction is given here to discrete internal representation schemes and their investigation by a pattern-perturbation technique. A more detailed account is given in Foster (1980a, b). A discrete internal representation is characterized by a finite set of finite subsets of basis elements, each subset associated with a finite number of attributes and attribute values. The set $A$ of all attributes can be partitioned into two subsets, $A^{D}$ and $A^{C}$. The subset $A^{D}$ consists of discrete attributes that can only assume values in a countable set equivalent to $\{1,2, \ldots, M\} \subset \mathbf{N}$, where $\mathbf{N}$ is the set of positive integers and $M$ depends on the attribute. The subset $A^{C}$ consists of continuous attributes that can only assume values in some interval $\mathbf{I}$ in the Euclidean space $\mathbf{R}^{l}$ where $\mathbf{R}$ is the set of real numbers and $\mathbf{I}$ and $l$ also depend on the attribute. An example of a continuous attribute associated with a basis element denoting a line in the visual field is the attribute designating the length of the line. An example of a discrete attribute associated with basis elements denoting a point and a fixed non-vertical non-intersecting line in the visual 
field is the attribute designating their vertical ordering, that is, whether the point is above or below the line. The finite set $X$ of basis elements and the finite set $A$ of attributes together constitute a fixed and finite repertoire of components from which an internal representation is considered to be constructed.

An important assumption in this scheme is that the selection of basis-element subsets, attributes, and attribute values for an internal representation is a probabilistic process. Suppose that two arbitrary basiselement subsets $\mathbf{x}_{i}$ and $\mathbf{x}_{j}$ are constituents of an internal representation and suppose that discrete attributes $\delta_{i \mu}, \mu=1,2, \ldots k(i)$, and continuous attributes $\gamma_{j v}, v=1,2, \ldots k^{\prime}(j)$, are associated with $\mathbf{x}_{i}$ and $\mathbf{x}_{j}$ respectively. Let $p_{i u}$ be the conditional discrete probability density function of $\delta_{i \mu}$ and let $f_{j v}$ be the conditional continuous probability density function of $\gamma_{j v^{*}}$. It was suggested in Foster (1980a) that if $B_{1}$ and $B_{2}$ are two subpatterns in the visual field, sufficiently alike in shape, then the discriminability of $B_{1}$ and $B_{2}$ is determined by the differences in the density functions $p_{i \mu}$ and in the density functions $f_{j v}$ for the two subpatterns. To explore the effect of these differences in density functions, patterns are drawn from a smooth continuum generated from a given (initial) pattern by the action of a local 1-parameter group of local transformations $\psi_{s}, s \in J$, where $J=[a, b]$ is an interval in $\mathbf{R}$ (see Appendix for definitions). This method ensures a transformational uniformity for the continuum (Foster, $1980 b$ ). Thus, if $\psi_{s}, s \in J$, is a local 1-parameter group of local transformations and $\Delta s \in J$, the change from $\psi_{s}$ to $\psi_{s+\Delta s}$ is the same as the change from $\psi_{s^{\prime}}$ to $\psi_{s^{\prime}+\Delta s^{\prime}}$, namely $\psi_{\Delta s}$. Let $\boldsymbol{B}$ be the initial pattern and let $\psi_{s}(B)$ be a transformed pattern. Suppose that for all $s \in J$ the transformed patterns $\psi_{s}(B)$ are such that only the probability density functions $p_{i \mu}$ and $f_{j v}$ of the attributes $\delta_{i \mu}$ and $\gamma_{j v}$ vary with $s$. For $\psi_{s}(B), p_{i \mu}(s, m)$ is the probability that the attribute $\delta_{i \mu}$ assumes the value $m$ and, for $U \subset \mathbf{R}^{l}, \int_{U} f_{j v}(s, u) d u$ is the probability that $\gamma_{j v}$ assumes a value in $U$. Suppose that $p_{i \mu}(s, \cdot)$ and $f_{j v}(s, \cdot)$ vary smoothly and continuously with $s$. Suppose further that $p_{i u}(s, \cdot)$ and $f_{j v}(s, \cdot)$ are such that the following conditions are satisfied.

(C1) For all discrete attributes $\delta_{i \mu}$ except for the $h \lambda^{\text {th }}$ the probability density functions $p_{i \mu}(\mathrm{s}, \cdot)$ are constant with $s$ and for all continuous attributes except the $k \underline{Q}^{\text {th }}$ the probability density functions $f_{j v}(s, \cdot)$ are constant with $s$. This condition is not essential, but it simplifies the exposition.

(C2) For the discrete attribute $\delta_{h \lambda}$ there is, for each $m, 1 \leqq m \leqq M$, a single interval $\left[c_{m}, d_{m}\right] \subset J$, where $c_{m} \leqq d_{m}$, on which $p_{h \lambda}(\cdot, m)$ achieves its maximum value and such that on $\left[a, c_{m}\right] p_{h \lambda}(\cdot, m)$ is monotonic increasing and on $\left[d_{m}, b\right]$ it is monotonic decreasing, with at most one point of inflexion in each case. For all $m$, $d_{m} \leqq c_{m+1}$. This smoothness property is assumed to hold, mutatis mutandis, for $\gamma_{k_{\ell}}$ and each $u \in \mathbf{I}$. This condition ensures that the smooth fall-off with $s$ in the probability assignment of each attribute value is not irregular.

\section{Discrimination of Transformed Patterns}

Consider now how the discriminability of pairs of patterns differing by a small, constant change in shape varies over the continuum defined by $s$.

If pattern $\psi_{s}(B)$ is separately given incremental transformations $\psi_{-\Delta s}$ and $\psi_{\Delta s}, \Delta s>0, \Delta s$ fixed, then $\psi_{-\Delta s}\left(\psi_{s}(B)\right)=\psi_{s-\Delta s}(B)$ and $\psi_{\Delta s}\left(\psi_{s}(B)\right)=\psi_{s+\Delta s}(B)$, providing that $s, s-\Delta s, s+\Delta s$ are in $J$. Following Foster (1980b), we suppose that the visual discriminability of the pair of perturbed patterns $\psi_{s-\Delta s}(B)$ and $\psi_{s+\Delta s}(B)$ is determined by the difference terms measured with the $l_{p}$ and $L_{p}$ norms,

$$
\begin{aligned}
& e_{h \lambda}(s)=\left(\sum_{m=1}^{M}\left|p_{h \lambda}(s+\Delta s, m)-p_{h \lambda}(s-\Delta s, m)\right|^{q}\right)^{1 / q}, \\
& e_{k \varrho}(s)=\left(\int_{1}\left|f_{k \varrho}(s+\Delta s, u)-f_{k \varrho}(s-\Delta s, u)\right|^{q^{\prime}} d u\right)^{1 / q^{\prime}},
\end{aligned}
$$

where $1 \leqq q, q^{\prime} \leqq \infty$. Note that

$$
\begin{aligned}
& \left(\sum_{m=1}^{M}\left|p_{h \lambda}(s+\Delta s, m)-p_{h \lambda}(s-\Delta s, m)\right|^{q}\right)^{1 / q} \\
& \quad \leqq\left(\sum_{m=1}^{M}\left|p_{h \lambda}(s+\Delta s, m)\right|^{q}+\sum_{m=1}^{M}\left|p_{h \lambda}(s-\Delta s, m)\right|^{q}\right)^{1 / q} \\
& \quad \leqq\left(\left|\sum_{m=1}^{M} p_{h \lambda}(s+\Delta s, m)\right|^{q}+\left|\sum_{m=1}^{M} p_{h \lambda}(s-\Delta s, m)\right|^{q}\right)^{1 / q} \\
& \quad=2^{1 / q} .
\end{aligned}
$$

That is, $\max e_{h \lambda}(s) \leqq 2^{1 / q}$. In fact, it is easy to show that $\max e_{h \lambda}(s)=2^{1 / q}$. Obviously, $\min e_{h \lambda}(s)=0$. The same results hold for $e_{k \rho}(s)$. If $q=1$ and $q^{\prime}=1$, the "cityblock" metric, the formulas (1) and (2) reduce to

$$
\begin{aligned}
& e_{h \lambda}(s)=2-2 \Sigma_{m=1}^{M} \min \left\{p_{h \lambda}(s+\Delta s, m), p_{h \lambda}(s-\Delta s, m)\right\}, \\
& e_{k \varrho}(s)=2-2 \int_{\mathrm{I}} \min \left\{f_{k \varrho}(s+\Delta s, u), f_{k \varrho}(s-\Delta s, u)\right\} d u .
\end{aligned}
$$

In the subsequent computations we shall always suppose $q=1$ and $q^{\prime}=1$. The discriminability $d^{\prime}$ of the pair of patterns $\psi_{s+\Delta s}(B), \psi_{s-\Delta s}(B)$ is assumed to be given by

$$
d^{\prime}(s)=y_{h \lambda}\left(w_{h \lambda} e_{h \lambda}(s)\right)+y_{k \varrho}\left(w_{k \varrho} e_{k \varrho}(s)\right),
$$

where $w_{h \lambda}$ and $w_{k \rho}$ are weighting coefficients depending on the selection probabilities of the attributes $\delta_{k \lambda}$ and $\gamma_{k e}$ and basis-element subsets $\mathbf{x}_{i}$ and $\mathbf{x}_{j}$, and where the functions $y_{h \lambda}$ and $y_{k e}$ are monotonic. The discriminability $d^{\prime}$ is the discrimination index as used in signal detection theory (Green and Swets, 1966; Swets, 1973), and has additivity properties made precise by Braida and Durlach (1972). Because we are dealing with one continuous attribute and one discrete attribute, we may simplify the notation. Thus set $e_{d}(s)=e_{h \lambda}(s)$, $e_{c}(s)=e_{k \rho}(s)$, where the subscripts $d$ and $c$ refer to 
discrete and continuous, and, since there is no risk of confusion, $\quad p(s, m)=p_{h \lambda}(s, m)$ and $f(s, u)=f_{k \varrho}(s, u)$. Furthermore we define

$$
\begin{aligned}
& d_{d}^{\prime}(s)=y_{h \lambda}\left(w_{h \lambda} e_{h \lambda}(s)\right), \\
& d_{c}^{\prime}(s)=y_{k \varrho}\left(w_{k \varrho} e_{k \varrho}(s)\right) .
\end{aligned}
$$

\section{Characterization of Discrimination Performance}

Several studies support the hypothesis that there is a limited pattern encoding capacity for the visual system (Estes and Taylor, 1964, 1966; Eriksen and Eriksen, 1974; Lupker and Massaro, 1979). If the number of patterns and subpatterns in a short duration display approaches this limit an internal representation containing discrete attributes should be more economical than one containing continuous attributes, for in a formal sense less information is specified by a discrete attribute than by a continuous attribute. The discrimination index $d^{\prime}$ should then depend solely on discrete attributes. Conversely, if a few subpatterns only are presented and viewing is prolonged it may be supposed that discrimination is determined solely by continuous attributes. Thus, in the former case $d^{\prime}(s)=d_{d}^{\prime}(s)$ and in the latter case $d^{\prime}(s)=d_{c}^{\prime}(s)$. The hypothesis that the number of subpatterns in the visual field determines the relative weighting of discrete and continuous attributes is analogous to a suggestion by Beck (Beck, 1972; Beck and Ambler, 1972, 1973) concerning the effect of subpattern number on "distributed" and "focal" attention in pattern discrimination. The simplest and most extreme case involving a single discrete attribute appears when the following condition is satisfied (see Foster, 1980b).

(C3) For adjacent attribute values $m-1, m, m+1$, $1<m<M$, the assignment probability of $m$ is not significant in either magnitude or relative rate of change when the pattern is transformed to or beyond the point at which $m-1, m+1$ have maximum assignment probabilities. For each transformed pattern $\psi_{s}(B)$, there are thus effectively at most two values, depending on $s$, of the discrete attribute that may be considered to be "in competition" with each other. If this condition is satisfied, then $p(s, m)=1-p(s, m-1)$ and Eq. (1) reduces to

$e_{d}(s)=2|p(s+\Delta s, m)-p(s-\Delta s, m)|$.

This case may be schematized in the following way, providing the smoothness constraint on $p(\cdot, m)$ is dropped. Given $s_{0} \in J$ and $\Delta s$ fixed, let $p(s, m)=1$ for $s \leqq s_{0}-\Delta s, p(s, m)=0$ for $s \geqq s_{0}+\Delta s$, and $\partial p(s, m) / \partial s=k$, where $k=-1 / 2 \Delta s$, for $s_{0}-\Delta s<s<s_{0}+\Delta s$. Obviously $p\left(s_{0}, m\right)=1 / 2$. The corresponding values of $e_{d}(s)$ are $e_{d}(s)=0 \quad$ if $\quad s \leqq s_{0}-2 \Delta s, \quad e_{d}\left(s_{0}-\Delta s\right)=1, \quad e_{d}\left(s_{0}\right)=2$,
$e_{d}\left(s_{0}+\Delta s\right)=1, e_{d}(s)=0$ if $s \geqq s_{0}+2 \Delta s$, and the maximum value of $e_{d}(s)$ is at $s=s_{0}$. The sharpness of this maximum may be quantified by means of the second divided difference $\Delta^{2} e_{d}(s)$, defined, for example, by the Newton form. After normalizing the scale of $s$ with respect to $\Delta s$, we obtain

$\Delta^{2} e_{d}\left(s_{0}\right)=\left(e_{d}\left(s_{0}-1\right)-2 e_{d}\left(s_{0}\right)+e_{d}\left(s_{0}+1\right)\right) / 2 ;$

that is,

$\Delta^{2} e_{d}\left(s_{0}\right)=-1$.

From this we suggest that $\min \Delta^{2} e_{d}(s)=-1$ should represent the archetypal discrete discrimination process.

For continuous attributes, it is reasonable to suppose that, for a given attribute value $u$, the function $f(\cdot, u)$ is different from zero only in a short subinterval of $J$. If $h$ is the distance between the two inflexion points of $f(\cdot, u)$, we can assume that $h \ll \Delta s$, which implies that for all $s$ in $J$ either $f(s+\Delta s, u)$ or $f(s-\Delta s, u)$ or possibly both are very small, ideally zero. Hence, from (4), $e_{c}(s)=2$. Therefore, $\Delta^{2} e_{c}(s)=0$, for all $s$, and this should represent the archetypal continuous discrimination process. ${ }^{1}$

\section{Empirical Values}

Results from the previous section concern the difference terms $e_{d}(s)$ and $e_{c}(s)$, and do not give predictions concerning the experimental values of the indices $d_{d}^{\prime}(s)$ and $d_{c}^{\prime}(s)$ which depend on $e_{d}(s)$ and $e_{c}(s)$ (see (5) and (6)). It may be noted, however, that the result $e_{c}(s) \geqq e_{d}(s)$ for all $s$ is not inconsistent with experimental findings, suggesting that the level of discrimination performance involving continuous attributes is greater than that involving discrete attributes (Foster, 1982, 1983). Nevertheless, it is possible to obtain a criterion to characterize discrete and continuous discrimination performances using the qualitative features of $d_{d}^{\prime}(s)$ and $d_{c}^{\prime}(s)$ resulting from definitions (5) and (6). If $e_{d}(s)$ has a maximum at a point $s=s_{0}$, then $d_{d}^{\prime}(s)$ must have a maximum at $s=s_{0}$ because of the monotonic relationship. Furthermore, it is obvious that if $e_{c}(s)$ is constant over all $s$ then $d_{c}^{\prime}(s)$ is constant. Thus, in principle, $\left(\min \Delta^{2} d_{c}^{\prime}(s)\right) /\left(\min \Delta^{2} d_{d}^{\prime}(s)\right)=0$.

1 It should be emphasized that the possibility of observing continuous or discrete modes of visual processing depends on the value assigned to the increment $\Delta s$. If $\Delta s$ is so large that the distance $h$ between inflexion points of $p(\cdot, m)$ is such that $h \ll \Delta s$ then, even though visual attention is distributed over several subpatterns in a display of short duration, discrimination may still be smooth with $s$. Conversely, if $\Delta s$ is so small that for $f(\cdot, u)$ the distance $h \simeq \Delta s$, then discrimination may appear non-smooth in experimental conditions in which attention may be distributed over few subpatterns in a display of long duration. A lower bound for the value of $\Delta s$ may be provided by the limit on visual acuity 
Table 1

\begin{tabular}{lcll}
\hline & Type I & Type II & Quotient \\
\hline 3-dot figures & -0.52 & -0.03 & 0.06 \\
Curved lines & -0.33 & -0.04 & 0.12 \\
\hline
\end{tabular}

To test this rule, minima of the second divided difference $\Delta^{2} d^{\prime}(s)$ over $s$ were computed from data on discrimination in displays of 3-dot figures (Foster, 1982) and on discrimination in displays of curved lines (Foster 1983). The design of these experiments was such that conditions $(\mathrm{C} 1)$ and $(\mathrm{C} 2)$ were satisfied and (C3) was not seriously violated. In each study, two kinds of discrimination experiment were performed: in the one, four subpatterns were presented in a display and duration was short $(100 \mathrm{~ms})$; in the other, two subpatterns were presented and duration was long ( $2 \mathrm{~s})$. In the first type of experiment, it is assumed that $d^{\prime}(s)=d_{d}^{\prime}(s)$ and in the second that $d^{\prime}(s)=d_{c}^{\prime}(s)$. Note that the use of $d^{\prime}$ allowed discrimination performance to be compared in different multi-alternative forcedchoice (here $2 \mathrm{AFC}$ and $4 \mathrm{AFC}$ ) paradigms. The results of these computations are shown in Table 1 where data from experiments with four subpatterns, $100-\mathrm{ms}$ duration, are labelled type I and those with two subpatterns, 2-s duration, are labelled type II. The quotients, 0.06 for the 3-dot figures and 0.12 for the curved lines, although not zero, are each acceptably small.

\section{Summary and Conclusion}

The framework for this study on discrete and continuous modes of visual pattern processing is based on the scheme for the description and investigation of discrete internal representations formulated by Foster (1980a, 1980b). This scheme entailed the use of a local 1-parameter group of local transformations $\psi_{s}, s \in J$, to obtain, from a given pattern $B$, a continuum of patterns $\psi_{s}(B), s \in J$, from which pairs of perturbed patterns $\psi_{s+\Delta s}(B), \psi_{s-\Delta s}(B)$ were selected and their discriminability measured as a function of the parameter $s$. In this paper, discrete and continuous modes of visual discrimination performance have been further analyzed to obtain a quantitative criterion typifying the two kinds of performance. A fundamental assumption has been that the contribution of discrete and continuous pattern attributes to performance depends on the number of subpatterns present in the visual field and on display duration, so that it is possible to hypothesize that for some experimental conditions only discrete attributes and for others only continuous attributes are selected. The smoothness or otherwise of the discrimination index $d^{\prime}$ as a function of $s$ is thus determined either by the characteristics of the difference term $e_{c}(s)$ or by the characteristics of the difference term $e_{d}(s)$. Since the presence of maxima is a qualitative feature of $e_{d}(s)$ that is independent of the monotonic scale transformation taking $e_{d}(s)$ to $d_{d}^{\prime}(s)$, it is plausible to use the ratio $\left(\min \Delta^{2} d_{c}^{\prime}(s)\right) /\left(\min \Delta^{2} d_{d}^{\prime}(s)\right)$ as a criterion to typify the relationship of the two kinds of performance.

Finally it may be observed that although this analysis has been developed within a particular framework, the use of the second divided difference to typify continuous and discrete discrimination performance can be extended to other kinds of experimental paradigm providing that performance has been quantified by a measure, such as $d^{\prime}$, that allows direct comparison of different multi-alternative forced-choice paradigms.

\section{Appendix}

Let $V$ be a subset of the plane $\mathbf{R}^{2}$ (or of $\mathbf{R}^{3}$ ) and let $J$ be an interval $[a, b]$ in $\mathbf{R}$, where $a<0, b>0$. A local 1-parameter group of local transformations defined on $V$ is a smooth mapping $\psi$ of $J \times V$ into $\mathbf{R}^{2}$ such that

(i) for each $s \in J, \psi_{s}=\psi(s, \cdot)$ is a smooth mapping (with smooth inverse) of $V$ onto $\psi_{s}(V)$;

(ii) if $s, s^{\prime}, s+s^{\prime} \in J$, and $z, \psi_{s^{\prime}}(z) \in V$, then $\psi_{s+s^{\prime}}(z)=\psi_{s^{\prime}}\left(\psi_{s^{\prime}}(z)\right)$;

(iii) for all $z \in V, \psi_{0}(z)=z$.

Acknowledgements. We thank S. R. Pratt and D. Lindsay for critical reading of the manuscript.

\section{References}

Beck, J. : Similarity grouping and peripheral discriminability under uncertainty. Am. J. Psychol. 85, 1-19 (1972)

Beck, J., Ambler, B.: Discriminability of differences in line slope and in line arrangement as a function of mask delay. Percept. Psychophys. 12, 33--38 (1972)

Beck, J., Ambler, B.: The effects of concentrated and distributed attention on peripheral acuity. Percept. Psychophys. 14, 225-230 (1973)

Braida, L.D., Durlach, N.I. : Intensity perception. II. Resolution in one-interval paradigms. J. acoust. Soc. Am. 51, 483-502 (1972)

Eriksen, B.A., Eriksen, C.W.: Effects of noise letters upon the identification of a target letter in a nonsearch task. Percept. Psychophys. 16, 143-149 (1974)

Estes, W.K., Taylor, H.A.: A detection method and probabilistic models for assessing information processing from brief visual displays. Proc. Natl. Acad. Sci. 52, 446-454 (1964)

Estes, W.K., Taylor, H.A.: Visual detection in relation to display size and redundancy of critical elements. Percept. Psychophys. 1, 9-16 (1966)

Foster, D.H.: Discrete internal pattern representations and visual detection of small changes in pattern shape. Percept. Psychophys. 26, 459-468 (1979)

Foster, D.H.: A description of discrete internal representation schemes for visual pattern discrimination. Biol. Cybern. 38, 151-157 (1980a)

Foster, D.H.: A spatial perturbation technique for the investigation of discrete internal representations of visual patterns. Biol. Cybern. 38, 159-169 (1980b) 
Foster, D.H.: The dependence of observer characteristics upon image complexity. In: IEE Conference Proceedings No. 214 "Electronic Image Processing". pp. 22-25. London: IEE 1982

Foster, D.H.: Visual discrimination, categorical identification, and categorical rating in brief displays of curved lines: implications for discrete encoding processes. J. Exp. Psychol. : Hum. Percept. Perform. 9, 785-806 (1983)

Fox, J.: Continuity, concealment and visual attention. In: Strategies of information processing. pp. 23-66. Underwood, G., (ed.). London: Academic Press 1978

Green, D.M., Swets, J.A.: Signal detection theory and psychophysics. New York, London, Sydney: Wiley 1966

Julesz, B.: Foundations of cyclopean perception. Chicago: University of Chicago Press 1971

Julesz, B.: Spatial nonlinearities in the instantaneous perception of textures with identical power spectra. Phil. Trans. Roy. Soc. Lond. B 290, 83-94 (1980)

Ludvigh, E.: Direction sense of the eye. Am. J. Ophthalmol. 36, 139-143 (1953)

Lupker, S.J., Massaro, D.W.: Selective perception without confounding contributions of decision and memory. Percept. Psychophys. 25, 60-69 (1979)
Olson, R.K., Attneave, F.: What variables produce similarity grouping? Am. J. Psychol. 83, 1-21 (1970)

Swets, J.A.: The relative operating characteristic in psychology. Science 182, 990-1000 (1973)

Westheimer, G.: The spatial sense of the eye. Invest. Ophthalmol. Visual Science 18, 893-912 (1979)

Westheimer, G.: Visual hyperacuity. In: Progress in sensory physiology. Vol. 1, pp. 1-30. Berlin, Heidelberg, New York: Springer 1981

Westheimer, G., McKee, S.P.: Spatial configurations for visual hyperacuity. Vision Res. 17, 941-947 (1977)

Received: October 28, 1983

Dr. David H. Foster

Dept. of Communication and Neuroscience

University of Keele

Keele/Staffordshire ST 55BG

England 